\title{
Design of an Ultrasonic Range Finder with DSP MSP430 Embedded System
}

\author{
ZHOU Hongfu ${ }^{1}$, FAN Qin ${ }^{2, *}$ \\ ${ }^{1}$ School of Mechanical and Automobile Engineering, South China University of Technology \\ ${ }^{2}$ School of Machinery and Automation, Wuhan University of Science and Technology \\ *Corresponding author, email : fanqin@wust.edu.cn (FAN Qin)
}

Keywords: Ultrasonic, distance measurement, range finder, MSP430

Abstract. This research designs an ultrasonic range finder where a MSP430F413 embedded system is used as a controller system. The research presents the instrument architecture with ultrasonic transmitted circuit, received circuit, embedded system. Also it study that the temperature and time interval error influences the distance detecting accuracy in the ultrasonic range finder,

\section{Introduction}

The ultrasonic distance measurement uses piezoelectric effect method to transmit ultrasonic signal in air by alternating voltage signal to incentive piezoelectric wafer for vibration, and receive the return ultrasonic signals for distance detection and analysis [1,2].

In the earlier time, Langevin, uses the low-frequency ultrasound to detect submarine underwater. In 1929, Sokolov studied the metal flaw detection with ultrasonic sound first time. In 1931, Mulhauser, German, filed a patent on ultrasonic range finder [3].

There are three kinds of ultrasonic distance detect methods, which are phase detecting, amplitude detecting and transiting time method. In this research, it uses the transiting time method for calculating the distance by detecting the ultrasonic passing time intervals from transmitting probe to receiving probe.

The applications of ultrasonic distance detecting include:

(1) In industry application, it includes position detection, mobile robot distance detecting and mobile robot obstacle avoidance, reverse collision checking.

(2) In the underwater detection, it can be used for underwater topography detection, and the seabed terrain detection.

(3) UAV landing detecting.

At present there are some researches in ultrasonic range finder:

(1) In material research of ultrasonic transducer, it effects on the precision of ultrasonic distance measurement [4]. In material selection, the transducers are fabricated by piezoelectric and magnetostrictive materials. But these two kinds of material are easy to suffer overshoot and cause measurement error.

(2) The research in improvement of measuring precision and detecting scope.

\section{The principle of ultrasonic measuring distance with time intervals}

The measuring working principle shows in Fig.1 and Fig.2, formula 1 is the measuring distance formula.

$\mathrm{S}=\mathrm{c} \times \mathrm{T} / 2$

In formula 1 and Fig.2, T, the total time, includes both transmitting and receiving time for the ultrasound signal. The distance detecting time is the half of the total time, $\mathrm{T} / 2$, and the $\mathrm{S}$ is the actual distance.

The $\mathrm{c}$ is for ultrasonic velocity, where it propagates in solid, liquid and gas, c, show in Formula 2.

$$
c=\sqrt{\mathrm{E} / \rho}
$$


In formula $2, \mathrm{E}$, the elastic modulus of medium, the unit is in $\mathrm{kg} / \mathrm{mm}^{2} ; \rho$, medium density, $\mathrm{kg} / \mathrm{mm}^{3}$; $\mathrm{c}$, the ultrasonic wave propagation velocity, unit in $\mathrm{m} / \mathrm{s}$.

From formula 2, the ultrasonic wave propagation velocity is related to the elastic modulus and density, where the density is affected by temperature in air propagation, the table 1.

In table 1 , if the ultrasonic wave propagation velocity select in $\mathrm{c}=344(\mathrm{~m} / \mathrm{s})$ at $20^{\circ} \mathrm{C}$, the time from transmitting to receiving the ultrasonic signal, it can be used as measuring the actual distance, $\mathrm{S}=344 \times \mathrm{T} / 2$ from formula 1 .

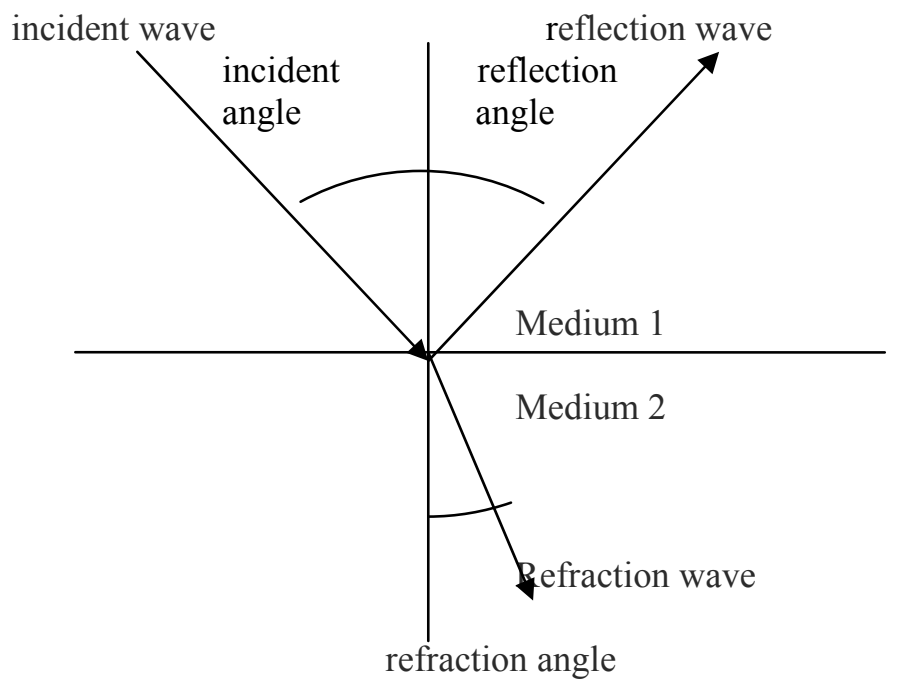

Fig.1. reflection of ultrasonic

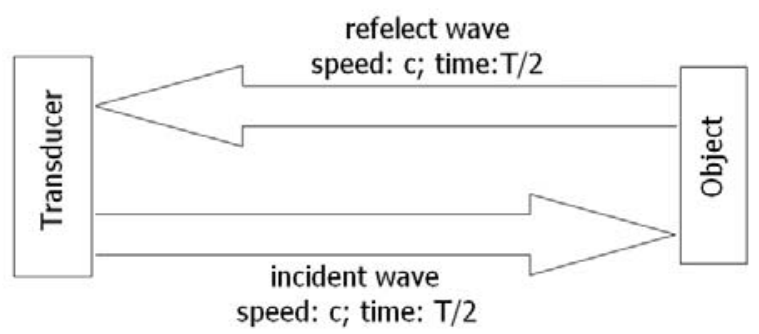

Fig.2. Ultrasonic range finder principle

Table 1 relation of ultrasonic wave propagation velocity with temperature

\begin{tabular}{l|l|l|l|l|l|l}
\hline Temperature $\left({ }^{\circ} \mathrm{C}\right)$ & -20 & -10 & 0 & 10 & 20 & 30 \\
\hline Speed $(\mathrm{m} / \mathrm{s})$ & 319 & 325 & 323 & 338 & 344 & 349 \\
\hline
\end{tabular}

\section{Hardware design}

\subsection{Ultrasonic distance detecting based on MSP430F413}

The system hardware characters are as followings:

Measuring range: $3 \mathrm{~m}$ below

Accuracy of measurement: under $5 \mathrm{~mm}$

Display: LED

Embedded system: TI MSP430, 64 pins QFP package, 16-bit RISC system

Fig. 3 shows the overall designing circuit block.

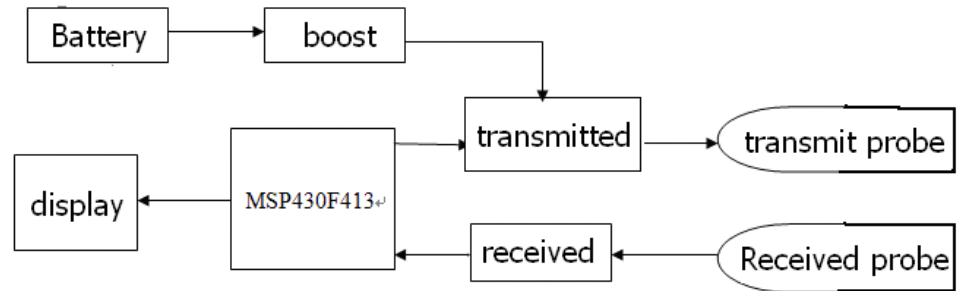

Fig.3. Circuit design block diagram 


\subsection{Embedded microcontroller}

This design uses two AA batteries for power supplier and power connect to CD4049 chip by a booster circuit. The embedded system, which shows in Fig.4, transmits $40 \mathrm{KHz}$ ultrasonic signal to detecting objects by transmitting probe, processes the receiving probe detecting signal, and displays the signal by the LED circuit.

The $40 \mathrm{KHz}$ square wave signal generates from the MSP430F413, Y1, crystal oscillator, and after amplifying it generates an ultrasound signal to transmitting probe.

The power supplier for MSP430F413 is 3.6 voltage, and diode D1 reverses connection for preventing damage of excessive current circuit.

In Fig. 4, P1.5 pin, it's for embedded system ACLK signal, with $40 \mathrm{KHz}$ square-wave. The voltage 3.3V connected to the OP-3.3VCC. At the same time, the AGnd \& Gnd connect together.

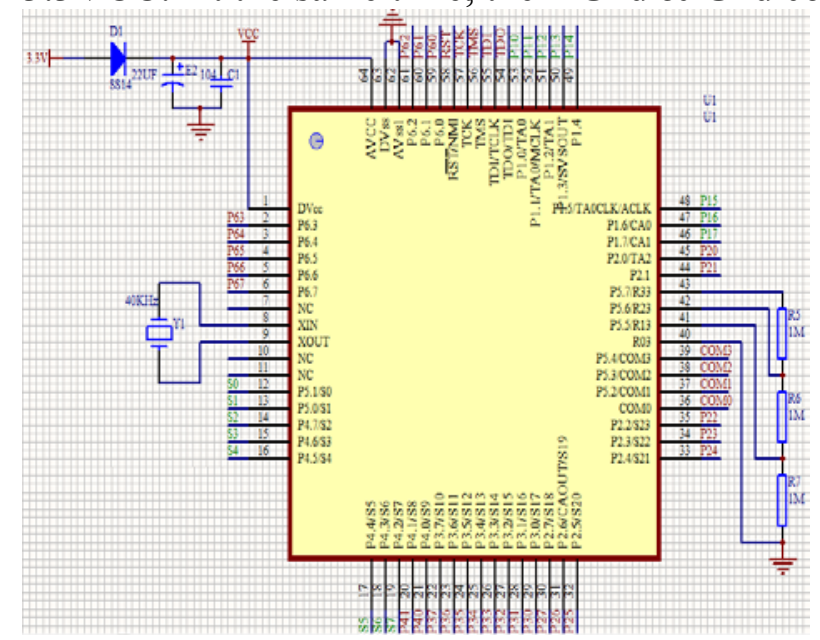

Fig.4. MSP430F413 embedded system

\subsection{Transmitting and receiving circuit}

The transmitted and received circuit show in Fig. 5.

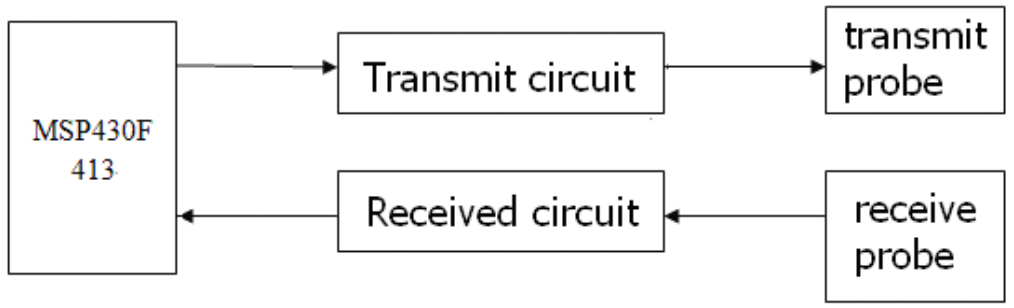

Fig.5. the block of distance detecting

Transmitting probe sends $40 \mathrm{KHz}$ ultrasonic pulse signals to the measuring object, and receiving probe detects the return signal for analysis the distance. Fig. 6 is the transmitting circuit, where the 40 $\mathrm{KHz}$ ultrasonic signal from embedded microcomputer to CD4049, makes the transmitting probe to resonance output.

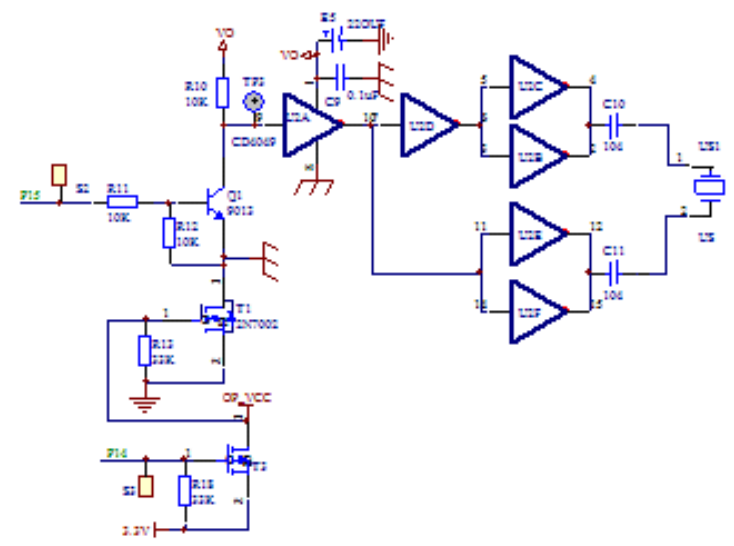

Fig. 6. Transmitting circuit

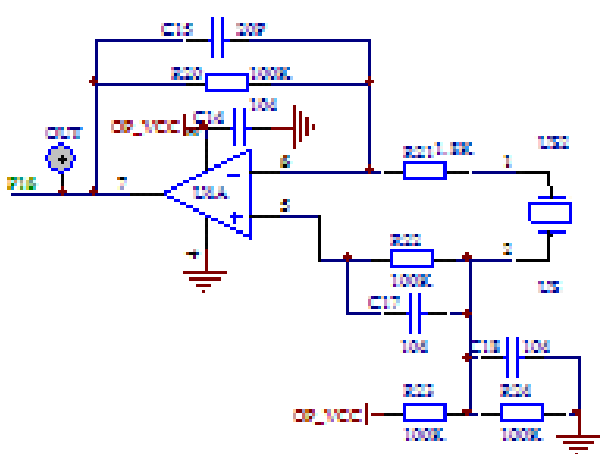

Fig. 7. Receiving circuit 
The receiving circuit shows in Fig. 7. In Fig.7, R20, R21 is used as to set the op-amp gain, and R23 and R24 for offset the inverting input.

\subsection{Boost Circuit}

The amplifier circuit shows in Fig. 8. MC34063 is a monolithic DC/DC conversion circuit, inside with DC-DC transform module. These functions mainly include, temperature compensation with the reference voltage source, the comparator, drives, large current output switch, etc.. The circuit is with less peripheral IC chips.

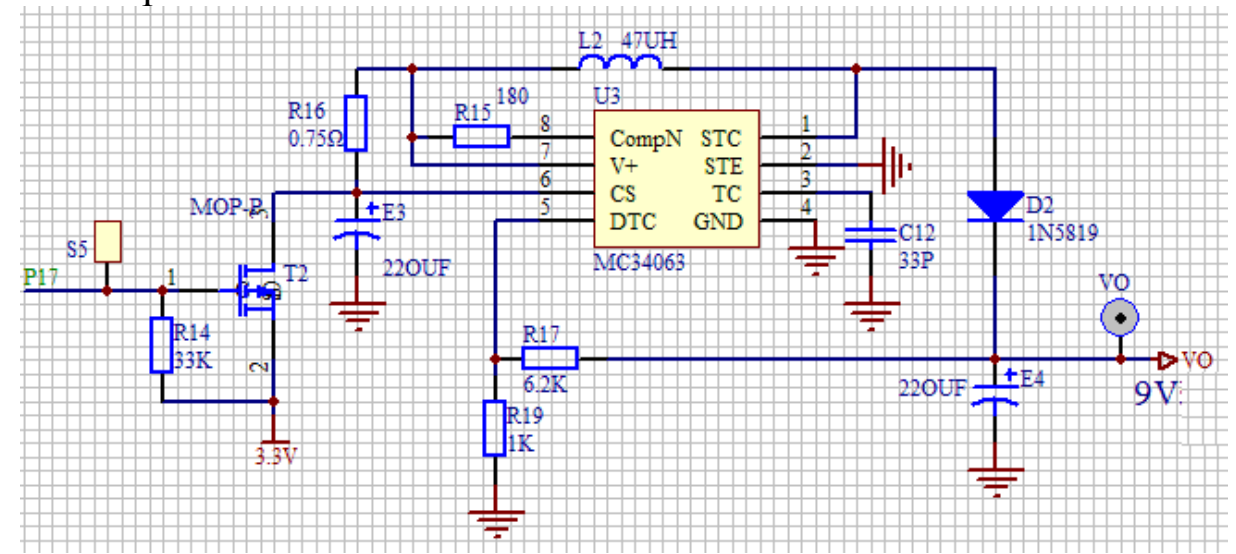

\section{5 reset circuit}

Fig.8. Boost Circuit

Reset circuit shows in Fig.9, where diode D3 can quickly release the charge on the capacitor C8, to ensure that the reset signal in stable. The reset unit can make the microcontroller reset to work from boost beginning.

\subsection{Display circuit}

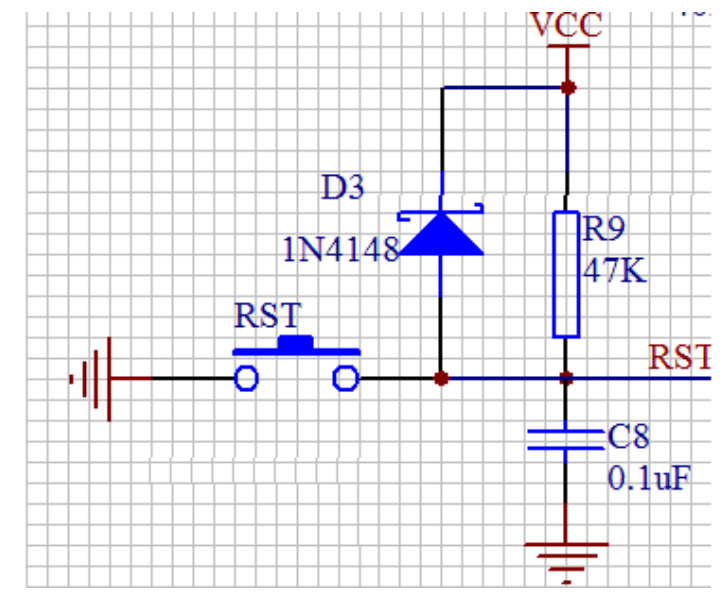

Fig.9. Reset Circuit

Fig.10 is the LED display circuit, where it uses 4 digits LED segment code for showing 4 decimal digit data. After processing the receiving ultrasonic signal, it displays the distance by the LED with the 4 decimal data.

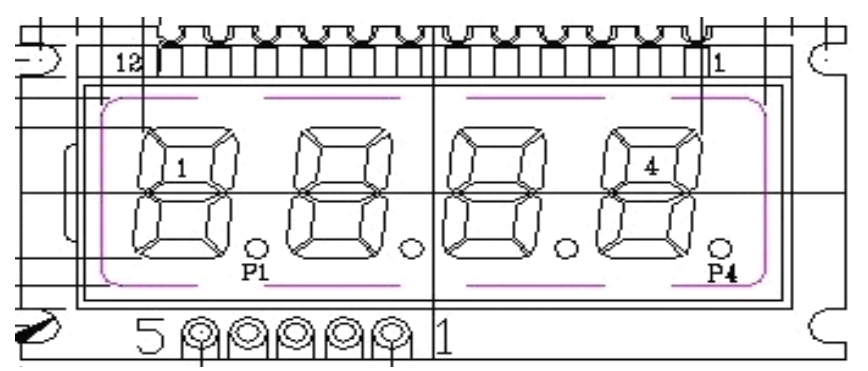

Fig.10. Sections of LED 


\subsection{Transducer}

Fig. 11 shows the transducer, which consists of two separating probes that shop from Taobao. In Fig. 11 , one transducer is for transmitting and the other for receiving.

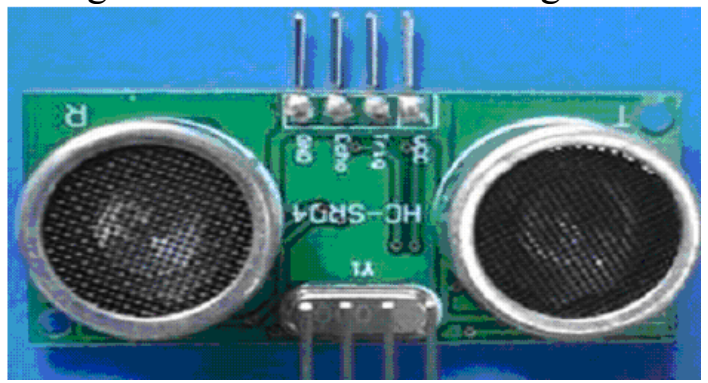

Fig.11. Transmitting and receiving probes
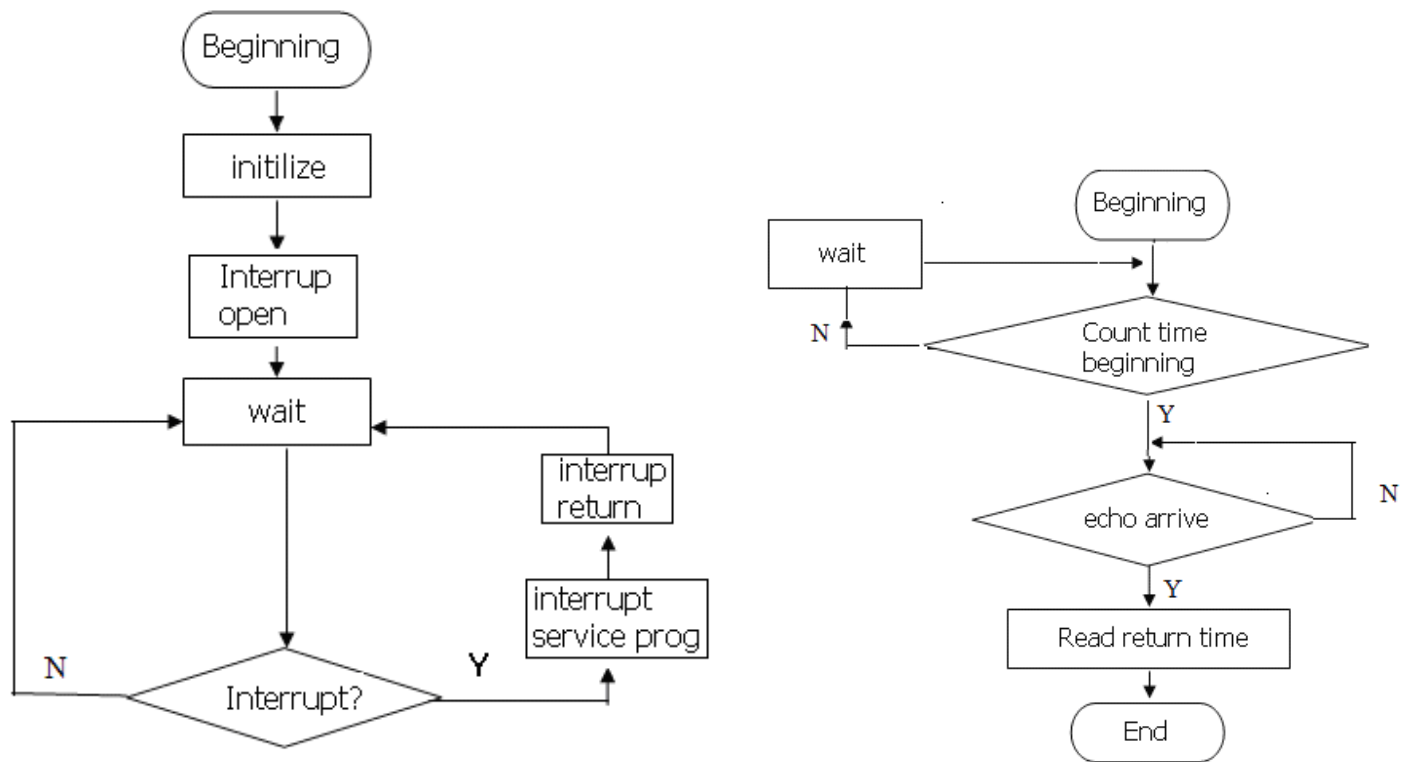

Fig.12. The main cycle program

Fig.13.Time interval check program

\section{Software design}

The ultrasonic range finder software is mainly composed of the time interval checking subroutine between transmitting and receiving, interrupting subroutine and displaying subroutine.

\subsection{Main cycle sub-program}

The main cycle sub-program includes the system environment initialization at first, such as MSP430F413 and LED initialization, interrupt trigger, and interrupt service sub-program, which shows in Fig. 12.

4.2 Time interval checking sub-program

The time interval checking sub-program shows in Fig.13, where it checks the time interval of signal transmitting from the transmitted probe beginning to receiving probe ending.

\section{The time interval and temperature errors influence to the distance detecting accuracy}

The time interval method measures the time between ultrasonic transmitting and receiving, and the measuring distance is obtained by the formula 1. If the error of the ultrasonic ranging is in a centimeter level, the measuring accuracy is relative to the accuracy of time interval and travelling velocity.

(1) The measuring time interval error. It affects the distance detecting error, it shows in formula 3. $\Delta \mathrm{S}=\Delta \mathrm{T}^{*} \mathrm{C} / 2$

From the formula 3 , if the measurement time error is less than $5.797 \mathrm{~ns}$, the measurement error is less than $1 \mathrm{~mm}$, where the average speed c values in $344 \mathrm{~m} / \mathrm{s}$ at $20.0{ }^{\circ} \mathrm{C}$. 
(2) The temperature error. According to the formula 2, ultrasonic transmission speed has relation with air density and elastic modulus, where the error of velocity, c, is affected by the air temperature $\mathrm{T}$, which shows in formula 4 . And the distance error compares to velocity error shows in formula 5.

$\mathrm{c}=\mathrm{c} 0+0.607 \mathrm{~T} \quad{ }^{0} \mathrm{C}$

$\Delta \mathrm{S}=\mathrm{S} * \Delta \mathrm{c} / \mathrm{c} \quad \mathrm{mm}$

In formula 4, where $\mathrm{c} 0$ is ultrasonic speed at $0{ }^{\circ} \mathrm{C}, \mathrm{T}$ is the actual air temperature, the unit in ${ }^{0} \mathrm{C}$.

From Table 1, the speed rate is in $323 \mathrm{~m} / \mathrm{s}$ at the air temperature of $0{ }^{\circ} \mathrm{C}$, and the speed rate is in 338 $\mathrm{m} / \mathrm{s}$ at 10 degrees Celsius. From the formula 4 and formula 5, for temperature error in 10 degrees Celsius in measuring the actual distance of $1 \mathrm{~m}$, the measurement error is about $18 \mathrm{~mm}$.

To reduce the measurement error caused by the error of temperature, it takes the temperature compensation, here it uses LM92 temperature sensor for temperature compensation. The LM92, a high precision temperature comparator sensor, it can compensate the temperature of the ultrasonic range finder. For measuring temperature, it uses LM35, an available temperature sensor and the sensitivity can reach $0.333{ }^{\circ} \mathrm{C}$, Fig. 14 .

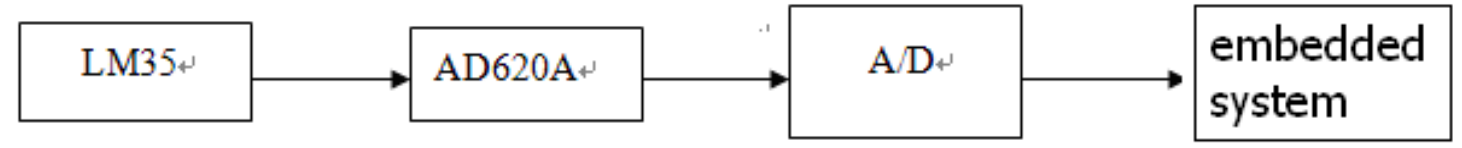

Fig.14. measurement of the environment temperature

\section{Summary}

The research designs an ultrasonic range finder device based on embedded system MSP430F413. It chooses $40 \mathrm{KHz}$ frequency as ultrasonic signal working frequency, and designs ultrasonic transmitted circuit and received circuit with DSP embedded system controlling. The design also presents an error calculating of the distance detecting method and it finds that the temperature and time interval error have a big influences to the distance detecting accuracy.

\section{References}

[1] Greg or io Andria, Filippo Attivissimo, Nico la Giaquinto. Digital signal processing techniques for accurate ultrasonic sensor measurement [J], Elsevier Science, 2001, 30:105-114

[2] J. H. Cantrcll, M. A. Bleazeale, Elimination of transducer Bond Corrections in Accurate Ultrasonic-wave velocity measurement by use of Capacitive Transducer [J]. The Journal of the Acoustical Society of America, Volume 61, Issue 2, February 1977, pp.403-406

[3] Karl F. Graff, A History of Ultrasonics, in Physical Acoustics, Volume XV (Academic Press, New York, 1982

[4] Ladabaum I, Jin X C, Soh H T. Surface micromachined capacitive ultrasonic transducers [J]. IEEE Trans on Ultrasonic, Ferroelectrics, and Frequency Control, 1998, 45( 3): 678-690 